\title{
Diagnosis and Treatment of Lambl's Excrescence on the Aortic Valve
}

\author{
Ren Chong-lei, MD, Jiang Sheng-li, MD, Wang Rong, MD, Xiao Cang-song, Wang Yao, Gao Chang-qing, MD \\ Department of Cardiovascular Surgery, Institute of Cardiac Surgery of PLA, Clinical Division of Surgery, Chinese PLA General \\ Hospital, Beijing 100853, China
}

\section{ABSTRACT}

Objective: Our objective is to summarize our experience in the diagnosis and treatment of Lambl's excrescence (LE) on the aortic valve.

Methods: The clinical data of 25 patients with LE admitted to our hospital from January 2010 to December 2014 were analyzed retrospectively. There were 17 males and 8 females, with the mean age of $55.7 \pm 11.43$ years (range: 30 to 70 years). Among the patients analyzed, eight also had cerebral embolism. All of the patients were diagnosed by transesophageal echocardiography (TEE). In seven cases, surgical treatment to remove the excrescence was successfully performed.

Results: All 25 patients were cured and discharged. There were no complications or operative mortalities in the seven patients that underwent surgical treatment. Followups were performed for all patients for an average of $2.9 \pm$ 1.5 years. During this time, none had a new cerebrovascular accident (CVA).

Conclusions: Most patients with LE are asymptomatic, though some patients showed repeated episodes of stroke. We recommend TEE as the main diagnostic means of the disease. Patients with LE that have experienced two or more CVAs or combined other heart disease and need open heart surgery should be offered surgical excision of the excrescences. Other patients should be treated conservatively with anticoagulation, or monitored closely.

\section{INTRODUCTION}

Aortic fibrous excrescence, also known as Lambl's excrescence (LE) and often referred to as valvular strands, is a fine $(\leq 2 \mathrm{~mm})$, thread-like ( $\geq 3 \mathrm{~mm})$ fibrotic valvular structure which occurs at the sites of valvular closure [Aziz 2007]. In 1856, Lambl's excrescence was first described by Dr. Vilém Dušan Lambl, who discovered the strands in $2 \%$ of autopsy cases. It was not believed to be clinically significant, therefore, little attention was paid to it. Following the development of advanced technology in clinical diagnosis, particularly the wide use of transesophageal echocardiogram (TEE), more aortic valvular strands have been identified. This has attracted

Received March 3, 2017; received in revised form December 8, 2017; accepted fanuary 13, 2018.

Correspondence: Ren Chong-lei, Department of Cardiovascular Surgery, Institute of Cardiac Surgery of PLA, Clinical Division of Surgery, Chinese PLA General Hospital, Beijing 100853, China; (e-mail: renchonglei301@163.com). increased attention, and debate over LE as a potential cause of stroke [Kalavakunta 2010; Armstrong 1997; Freedberg 1995]. In the current study, we retrospectively analyzed 25 patients with LE to summarize our experience in its diagnosis and treatment.

\section{METHODS}

The clinical data of 25 patients with LE who received treatments in our hospital from January 2010 to December 2014 were analyzed retrospectively (Table). There were 17 males and 8 females, with a mean age of $55.7 \pm 11.43$ years (range, 30 to 70 years). Among these patients, there were eight with cerebral embolism (32\%), two with coronary artery disease, two with rheumatic heart disease, and six with hypertension. All patients were evaluated by TEE alone, or additionally verified by surgery.

All LEs were diagnosed by TEE with GE Vivid 7 systems (GE Company, USA). Of the 25 cases, 12 were routinely examined by TEE for atrial fibrillation (AF) ablation, 8 were investigated by TEE in terms of cause of stroke, and the other 5 were inspected by further TEE evaluation due to suspected transthoracic echocardiography (TTE) findings. LE was defined as thin $(\leq 2 \mathrm{~mm})$, elongated $(\geq 3 \mathrm{~mm})$ structures with independent and undulating hypermobility, seen at the leaflet's coaptation [Freedberg 1995].

Surgical treatment was successfully performed on seven patients to remove the excrescences. The indications for surgery in patients with LEs include those with two or more cerebrovascular accidents (CVAs), and those who are undergoing concomitant heart surgery for other heart disease. We performed isolated LE excision on three patients. A concomitant coronary artery bypass graft (CABG) was performed on two patients, and a mitral valve replacement on two others. Surgery was performed under general analgesia through mid-sternotomy;

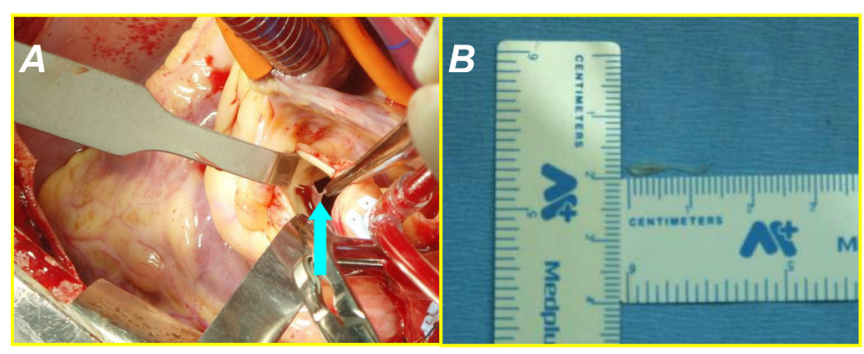

Figure 1. A) Lambl's excrescence during operation (indicated by arrow). B) Gross photo in vitro. 
Clinical Parameters in 25 Patients with LE on Aortic Valve

\begin{tabular}{lc}
\hline Parameter & Value or Case Number (\%) \\
\hline Male & $17(68 \%)$ \\
Age, years & $55.7 \pm 11.43$ \\
Complications & \\
Atrial fibril/flutter & $12(48 \%)$ \\
Cerebral infarction & $8(32 \%)$ \\
Hypertension & $6(24 \%)$ \\
Coronary heart disease & $2(8 \%)$ \\
Rheumatic heart disease & $2(8 \%)$ \\
First diagnosed disease & \\
Atrial fibril/flutter & $12(48 \%)$ \\
Cerebral infarction & $8(32 \%)$ \\
Coronary heart disease & $2(8 \%)$ \\
Rheumatic heart disease & $2(8 \%)$ \\
Health check & $1(4 \%)$ \\
Purpose of TEE & \\
Routine preoperative test & $12(48 \%)$ \\
Cardiogenic cerebral embolism & $8(32 \%)$ \\
Suspicious excrescences on TTE & $5(20 \%)$ \\
Excrescences location on TEE & $18(72 \%)$ \\
Left coronary cusp & $7(28 \%)$ \\
Right and no coronary cusp & \\
& \\
\hline
\end{tabular}

TEE indicates transesophageal echocardiography; TTE, transthoracic echocardiography.

cardiopulmonary bypass was achieved with bi-caval and aorta cannulation. The diagnosis was confirmed through intraoperative findings of fibrous stands on the free edge of the aortic valve (Figure 1A). The vegetation was completely excised (Figure 1B) from the base attached to the leaflets using fine scissors, without damaging the leaflets. Intraoperative TEE showed normal valve function with no aortic regurgitation.

Long term antiplatelet or warfarin therapy was prescribed for 19 patients after diagnosis or surgery. Three patients who received LE excision took only aspirin for three months postoperatively, and the other three patients were monitored closely without medication.

\section{RESULTS}

All 25 patients were uneventfully discharged. Preoperational TEE findings revealed isolated, mobile, thin, threadlike structures of $3-13 \mathrm{~mm}$ in length (Figures $2 \mathrm{~A}$ and $2 \mathrm{~B}$ ) located at the left coronary cusp (18 cases), right, and/or noncoronary cusp (7 cases).

There were no complications or operative mortality in the seven patients who underwent surgical excision.

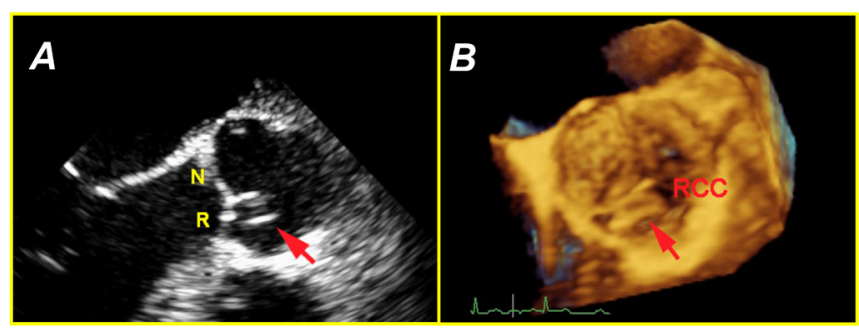

Figure 2. Lambl's excrescence on aortic valve. A) Transoesphageal echocardiography: a mobile, thin, thread like Lambl's excrescence on right coronary cusp (indicated by arrow). B) Three-dimensional echocardiography: Lambl's excrescence (indicated by arrow).

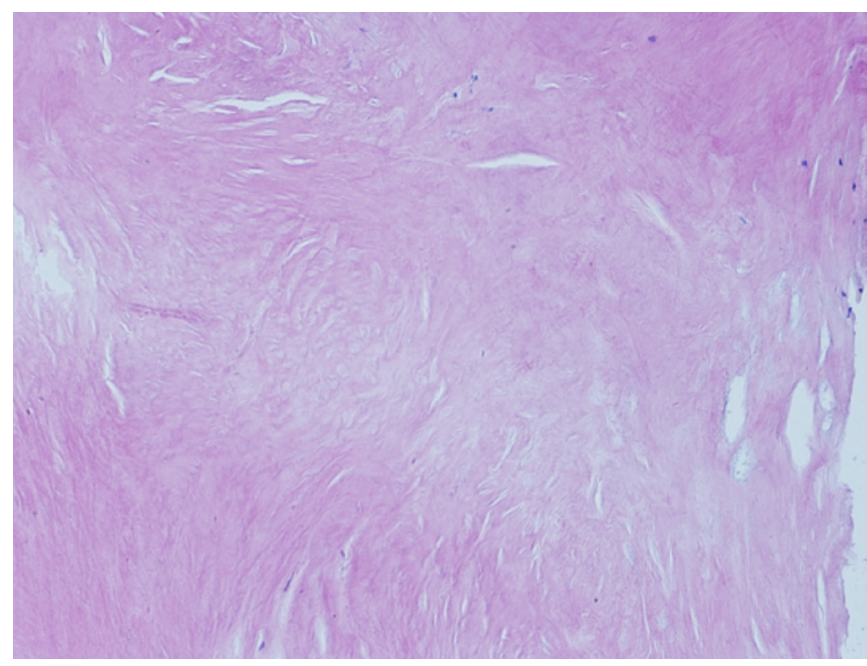

Figure 3. Photomicrography showing collagenous degeneration in Lambl's excrescence.

Intraoperative TEE results were consistent with the preoperative investigation. Visual observations confirmed the presence of visible fibrous filament on the free edge of the aortic valve during the operation. The LEs found during the operation were $3-13 \mathrm{~mm}$ long, and sited at the left (5 cases) or right coronary cusp (2 cases). Histopathological findings confirmed fibrous excrescence (Figure 3).

All 25 patients were followed up for an average of $2.9 \pm 1.5$ years (range, 1 to 5 years). During the follow up, no patient had a new cerebrovascular accident (CVA) or bleeding disorder. The seven patients that underwent surgical resection had no recurrence of the LE when echocardiographical follow up was performed.

\section{DISCUSSION}

LE is a thin, mobile, filiform structure, often referred to as valvular strands in echocardiography literature [Jaffe 2007]. These valvular strands may occur as a single strand, in rows, or in clusters. Histologically, theses excrescences are composed of a cellular connective tissue core covered by endothelium, which contains abundant collagen fibrils and elastic material. This tissue is arranged in apposed layers, with different 
collagen fibril orientation. These lesions may occur in association with chronic rheumatic heart disease, pulmonary or systemic hypertension, or old endocarditis, but may also be seen without any additional cardiac disease [Nighoghossian 1997]. Their pathogenesis remains uncertain and is believed to result from valvular wear and tear. Fibrin deposition over damaged endocardial valvular surfaces subsequently becomes partially detached from the valve, condensed and hyalinized, and ultimately fibrosed [Kalavakunta 2010; Aggarwal 2003; Nighoghossian 1997].

Most patients with LE are asymptomatic [Aziz 2007]. However, LE, found mostly on aortic valve leaflets can break apart and embolize. When they embolize to the brain, they can cause stroke. The potential cause of a CVA should be identified by Doppler ultrasonography of the carotid arteries and by echographical scanning of the ascending, transverse, and arch segments of the aorta [Jaffe 2007]. In our research, less than $1 / 3(8 / 25)$ of the symptomatic cases were identified during investigation for cardiogenic cause of stroke, and almost half $(12 / 25)$ of the cases were found with LE in the preoperative, routine TEE investigation. Five of the cases (20\%) were further studied with TEE evaluation, due to suspected TTE findings. We recommend TEE as a component in the causal evaluation of any stroke, because it is more sensitive than TTE [Leitman 2014].

On TEE, LE appears as an independent, hypermobile, thin, strand-like structure ( $\leq 2 \mathrm{~mm}$ in thickness and $\geq 3 \mathrm{~mm}$ in length) on the line of closure of the valves [Jaffe 2007; Roldan 1997]. The differential diagnoses of LE includes imaging artifact, vegetation, thrombus, redundant leaflet, flap due to aortic dissection, and papillary fibroelastoma [Jaffe 2007; Daveron 2005]. In contrast to LE, papillary fibroelastomas appear as pedunculated lesions on the less mechanically-affected parts of valves, and on other areas of the endocardium.

Because the association of $\mathrm{LE}$ with the risk of embolism is uncertain, treatment of LE remains controversial, and no corresponding guideline can be referred to. It is suggested that patients who have LE and have experienced one episode of CVA should be treated conservatively with anticoagulation, whereas the second CVA should lead to consideration of surgical excision of the LE [Nakahira 2008; Aziz 2007]. Asymptomatic patients should be monitored closely. Some believe that perhaps these filaments are simply "innocent bystanders" of no pathophysiologic significance, based on the fact that there may not be a direct causal link between ischemic stroke and the presence of the valve strands [Roldan 2015; Melduni 2008; Cohen 1997]. Therefore, it is recognized that there may be occasional exceptions, especially when large lesions with a head or a stalk are found [Melduni 2008]. Our follow up results showed the reliability and effectiveness of the LE's treatment, however, more cases with longer follow up periods are required to establish the final guidelines.

The limitations of this study were that it was a single-center retrospective observational study, and the small number of case samples, which may affect the power of the conclusions.
To establish guidelines or consensuses for LE treatment, a larger number of cases is still needed to provide evidence.

In this study, most patients with LE were asymptomatic, though some patients showed repeated episodes of stroke. We recommend TEE as the main diagnostic means of the disease. Patients who have LE and have experienced two or more CVAs or have another combined heart disease in need of an open heart surgery should be offered surgical excision of the excrescence, though other LE patients should be treated conservatively with anticoagulation or monitored closely.

\section{REFERENCES}

Aggarwal A, Leavitt BJ. 2003. Giant lambl's excrescences. N Engl J Med 349:25.

Armstrong WF. 1997. Valve excrescences: harmless and common or strokes-in-waiting? J AmColl Cardiol 30:1315-6.

Aziz F, Baciewicz, FA Jr. 2007. Lambl's Excrescences: review and recommendations. Tex Heart Inst J 34:366-8.

Cohen A, Tzourio C, Chauvel C, et al. 1997. Mitral valve strands and the risk of ischemic stroke in elderly patients. Stroke 28:1574-8.

Daveron E, Jain N, Kelley GP, et al. 2005. Papillary fibroelastoma and Lambl's excrescences: echocardiographic diagnosis and differential diagnosis. Echocardiography 22:285-7.

Freedberg RS, Goodkin GM, Perez JL, et al. 1995. Valve strands are strongly associated with systemic embolization: a transesophageal echocardiographic study. J Am Coll Cardiol 26:1709-12.

Jaffe W, Figueredo VM. 2007. An example of lambl's excrescences by transesophageal echocardiogram: a commonly misinterpreted lesion. Echocardiography 24:1086-9.

Kalavakunta JK, Peddi P, Bantu V, et al. 2010. Lambl's excrescences: a rare cause of stroke. J Heart Valve Dis 19:669-70.

Leitman M, Tyomkin V, Peleg E, et al. 2014. Clinical significance and prevalence of valvular strands during routine echo examinations. Eur Heart J Cardiovasc Imaging 15:1226-30.

Melduni RM, Klarich KW, Nesbitt GC, et al. 2008. Lambl's excrescences: is surgical excision really necessary? Tex Heart Inst J 35:89.

Nakahira J, Sawai T, Katsumata T, et al. 2008. Lambl's excrescence on aortic valve detected by transesophageal echocardiography. Anesth Analg 106:1639-40.

Nighoghossian N, Derex L, Loire R, et al. 1997. Giant lambl excrescences: an unusual source of cerebral embolism. Arch Neurol 54:41-4.

Roldan CA, Schevchuck O, Tolstrup K, et al. 2015. Lambl's excrescences: association with cerebrovascular disease and pathogenesis. Cerebrovasc Dis $40: 18-27$.

Roldan CA, Shively BK, Crawford MH. 1997. Valve excrescences: prevalence, evolution and risk for cardioembolism. J Am Coll Cardiol 30:1308-14. 